\title{
Orientational decoherence within molecules and emergence of the molecular shape
}

\author{
Edit Mátyus ${ }^{1, *}$ and Patrick Cassam-Chenaì ${ }^{2,+}$ \\ ${ }^{1}$ Institute of Chemistry, Eötvös Loránd University, \\ Pázmány Péter sétány 1/A, 1117 Budapest, Hungary \\ ${ }^{2}$ Université Côte d'Azur, CNRS, \\ LJAD, UMR 7351, 06100 Nice, France
}

(Dated: January 5, 2021)

\begin{abstract}
The question of classicality is addressed in relation with the shape of the nuclear skeleton of molecular systems. As the most natural environment, the electrons of the molecule are considered as continuously monitoring agents for the nuclei. For this picture, an elementary formalism of decoherence theory is developed and numerical results are presented for few-particle systems. The numerical examples suggest that the electron-nucleus Coulomb interaction is sufficient for inducing a blurred shape with strong quantum coherences in compounds of the lightest elements, $\mathrm{H}_{2}, \mathrm{D}_{2}, \mathrm{~T}_{2}$, and $\mathrm{HeH}^{+}$.
\end{abstract}

*Electronic address: matyus@chem.elte.hu

$\dagger^{\dagger}$ Electronic address: cassam@unice.fr 


\section{INTRODUCTION}

We would like to better understand the status of the permanent chemical observables by starting from a fully quantum mechanical description of a molecule, i.e., by including both the electrons and the atomic nuclei in the quantum treatment. The present work is concerned with the recognition of elements of the classical molecular shape. A fundamental difficulty is associated with the fact that the classical shape (unless spherically symmetric) breaks the rotational symmetry of the molecular wave function of an isolated molecule [1].

There exist quick shortcuts to this problem. First (case I), pieces of information regarding the molecular shape (and structure) can be obtained by fitting an effective model Hamiltonian to the isolated molecule's rotational spectrum [2]. Second (case II), in many chemical experiments the molecules are in some environment, and thus, the isolated-system symmetries are not longer relevant.

Nevertheless, we remain interested in the original theoretical problem, that had became famous as the molecular structure conundrum [3-9]: we consider an isolated molecule and aim to explore the furthermost point regarding the molecular shape (and structure) without considering any kind of an environment (as in case II) or making any a priori assumption on the hierarchical separation in the spatio-temporal behaviour of the internal dynamics to build model Hamiltonians (as in case I).

But we have said that the classical molecular shape breaks a fundamental symmetry of the isolated quantum system. So, how can we expect to see the emergence of a symmetry breaking feature without actually breaking the symmetry?

The core of this work rests on understanding what is quantum, what is classical, and how they are connected. We borrow tools from decoherence theory [10, 11] and use them for the molecular problem. Interference is one of the key concepts for understanding quantum behavior. If interference vanishes, then the quantum system has classical-like features. In practical terms, we have to consider the system's reduced density matrix (RDM). If its off-diagonal elements (interference terms) are suppressed in some representation, then the RDM is formally identical with that of a classical mixture (Fig. 11). Based on this mathematical equivalence, we may say that a quantum system resembles a classical statistical ensemble. Science philosophers 

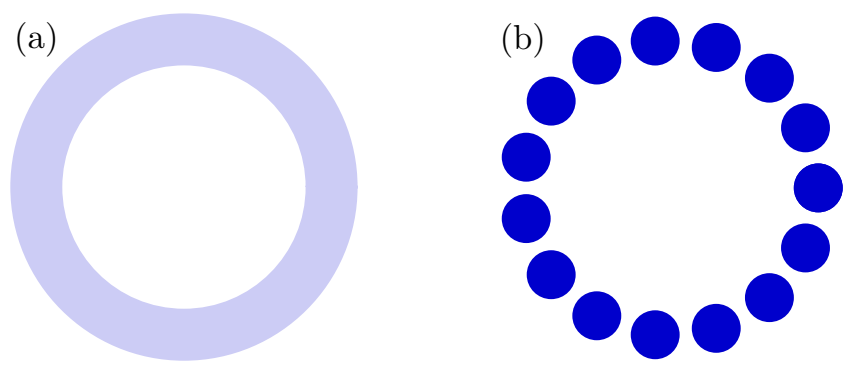

FIG. 1: Illustration of a rotationally symmetric object with (a) strong rotational interference and (b) vanishing rotational interference. If the interference vanishes (b), the quantum system resembles a collection of (rotated) classical objects.

[12, 13] as well as pioneers in decoherence theory [10] have noticed that suppression of interference between selected states is not a sufficient condition to fully reduce the quantum world to one amenable to a classical treatment. It is a necessary one. In the present work, we will follow this common and fruitful interpretation of the quantum-classical relation that is based on the suppression of the interference terms.

Pursuing decoherence ideas for the molecular problem is not new and has always started with the definition of an environment model. Pfeifer [14, 15] considered the vacuum state of the electromagnetic field as a general environment to model localization of enantiomers. This model was later debated for several reasons. Woolley argued [16] that Pfeifer's non-gauge invariant representation and linear approximation in the field operators resulted in an artificial localization. It was also shown that the proposed mechanism fails for non-zero temperature [17]. Zeh argued (p. 387 of Ref. [10]) that a non-trivial dressing (on its own) does not guarantee transformation from a superposition to an ensemble.

Independently, Claverie and Jona-Lasinio [18, 19] used external random noise to simulate localization in a double-well problem to model molecular chirality. Joos and Zeh studied simple scattering models [20], and Hornberger and co-workers simulated the stabilization of chiral molecules upon collisions [21-23]. More recently, change of the environment model parameters were studied on the localization of mesoscopic quantum objects [24]. A systematic and accurate calculation of the decoherence times for molecular processes in interaction with a series of environment models is very interesting and may turn out to be useful for designing better quantum computers (with molecular qubits). 
For solving the molecular structure problem, one would like to find the most general possible environment model for a molecule. When we think about the structure of a molecule, we rarely have to specify the corresponding environment. Most probably, this desire led Pfeifer in 1980 [14] to pick the electromagnetic vacuum state as an environment.

In the present work, instead of setting out to design the most general environment for a molecule, we will take a closer look at the molecular wave function. We have said that we did not want to introduce any a priori dynamical assumptions in the molecular quantum treatment. But when we have the full molecular wave function at hand, we can, of course, exploit the different properties of the particles during the course of an a posteriori analysis of the wave function. So, in the present work, we study the spatial coherence or decoherence of the nuclear skeleton induced by the continuous monitoring effect of the electron cloud. This information is encoded in the molecular wave function, and we will write down the formalism that makes this 'visible'.

The present study of spatial (de)coherence within the molecule adds a missing bit to our earlier understanding [25-30] that followed a path proposed by Claverie and Diner [5]. According to them, elements of the molecular structure can be recognized as nuclear configurations for which the particle density is large. Large values of the particle density certainly indicate configurations that are the most probable, but it does not tell us whether an assembly of particles have the classical-like features that we know about in chemistry.

In Sec. II we work out the basic decoherence formalism for spatial localization of nuclei in the molecule. Sec. III makes the formalism specific for the molecular shape and Sec. IV presents numerical results computed from molecular wave functions. 


\section{MOLECULAR DECOHERENCE THEORY}

When a property of a quantum system is measured, the needle of an idealized measuring device points to one of the possible outcome values. In an ideal quantum mechanical description of measurement, the (macroscopic) experimental setup should be included in a quantum treatment [6, 31]. The quantum states corresponding to the positions of the measuring needle were termed 'pointer states' by Zurek [32] and their apparent classical behaviour is ensured by the decoherence effect of the measuring environment.

Nowadays, the concept of pointer states is used in a more abstract sense without associating an actual experimental setup to them. In the pointer basis representation, the off-diagonal elements of the reduced density matrix, which represent quantum coherence between the states of the system associated to the environment pointer states, are suppressed.

In general, we do not know a priori the pointer states of a quantum system in a given environment. For each microscopic environment model, one has to find the proper pointer states that will point to the classical-like (environmentally stabilized)

states of the quantum system [22, 32]. Localization of macroscopic objects in space (e.g., translational localization) is studied in a so-called 'direct representation' using a set of Dirac delta distributions over the configuration space.

By molecular shape (and internal structure), we understand the localization of the nuclei in the three dimensional space. So, our 'measuring needles' are functions located in the three-dimensional space ('position basis'). Hence, we will be concerned with the 'direct representation' of the density matrix.

Let $|\Psi\rangle$ be a normalized molecular wave function. The associated (pure-state) density operator is denoted by $\hat{\rho}=|\Psi\rangle\langle\Psi|$. Let us multiply $\hat{\rho}$ both from the left and from the right with the resolution of identity written in the position basis of the electrons and the nuclei, $\chi_{r}$ and $\chi_{R}$, respectively. Hence, the molecular (pure-state) 
density operator is written as

$$
\begin{aligned}
\hat{\rho} & =|\Psi\rangle\langle\Psi| \\
& =\hat{I} \cdot|\Psi\rangle\langle\Psi| \cdot \hat{I} \\
& =\int \mathrm{d} r \mathrm{~d} R\left|\chi_{r} \chi_{R}\right\rangle\left\langle\chi_{r} \chi_{R}|\cdot| \Psi\right\rangle\left\langle\Psi\left|\cdot \int \mathrm{d} r^{\prime} \mathrm{d} R^{\prime}\right| \chi_{r^{\prime}} \chi_{R^{\prime}}\right\rangle\left\langle\chi_{r^{\prime}} \chi_{R^{\prime}}\right| \\
& =\int \mathrm{d} r \mathrm{~d} R \mathrm{~d} r^{\prime} \mathrm{d} R^{\prime}\left|\chi_{r} \chi_{R}\right\rangle\left\langle\chi_{r^{\prime}} \chi_{R^{\prime}}\right| \Psi(r, R) \Psi^{*}\left(r^{\prime}, R^{\prime}\right)
\end{aligned}
$$

Since we are interested in the nuclear structure, we integrate out the electronic degrees of freedom, and obtain the nuclear reduced density matrix,

$$
\begin{aligned}
\hat{\rho}_{\text {nuc }} & =\operatorname{Tr}_{\mathrm{el}}[\hat{\rho}] \\
& =\int \mathrm{d} r^{\prime \prime}\left\langle\chi_{r^{\prime \prime}} \mid \Psi\right\rangle\left\langle\Psi \mid \chi_{r^{\prime \prime}}\right\rangle \\
& =\int \mathrm{d} r^{\prime \prime}\left\langle\chi_{r^{\prime \prime}} \mid\left[\int \mathrm{d} r \mathrm{~d} R \mathrm{~d} r^{\prime} \mathrm{d} R^{\prime}\left|\chi_{r} \chi_{R}\right\rangle\left\langle\chi_{r^{\prime}} \chi_{R^{\prime}}\right| \Psi(r, R) \Psi^{*}\left(r^{\prime}, R^{\prime}\right)\right] \chi_{r^{\prime \prime}}\right\rangle \\
& =\int \mathrm{d} R \mathrm{~d} R^{\prime}\left|\chi_{R}\right\rangle\left\langle\chi_{R^{\prime}}\right| \int \mathrm{d} r^{\prime \prime} \Psi\left(r^{\prime \prime}, R\right) \Psi^{*}\left(r^{\prime \prime}, R^{\prime}\right),
\end{aligned}
$$

where the element of the reduced density matrix in the position representation is denoted as

$$
\rho_{\text {nuc }}\left(R, R^{\prime}\right)=\left\langle\chi_{R}\left|\hat{\rho}_{\text {nuc }}\right| \chi_{R^{\prime}}\right\rangle=\int \mathrm{d} r^{\prime \prime} \Psi\left(r^{\prime \prime}, R\right) \Psi^{*}\left(r^{\prime \prime}, R^{\prime}\right) .
$$

It is worth making explicit the diagonal and off-diagonal contributions to $\hat{\rho}_{\text {nuc }}$

$$
\begin{aligned}
\hat{\rho}_{\text {nuc }}= & \int \mathrm{d} R\left|\chi_{R}\right\rangle\left\langle\chi_{R}\right| \rho_{\text {nuc }}(R, R) \\
& +\int \mathrm{d} R \mathrm{~d} R^{\prime}\left|\chi_{R}\right\rangle\left\langle\chi_{R^{\prime}}\right|\left[1-\delta\left(R-R^{\prime}\right)\right] \rho_{\text {nuc }}\left(R, R^{\prime}\right),
\end{aligned}
$$

where the second term describes the interference between the nuclear structures at $R$ and $R^{\prime}$. If the $\rho_{\text {nuc }}\left(R, R^{\prime}\right)$ off-diagonal elements are small, then

$$
\hat{\rho}_{\text {nuc }} \approx \int \mathrm{d} R\left|\chi_{R}\right\rangle\left\langle\chi_{R}\right| \rho_{\text {nuc }}(R, R)
$$


that is mathematically equivalent with the density matrix of a classical mixture of localized structures at $R$ (a mixed state). According to decoherence theory and based on this mathematical equivalence, we may say that the quantum system has classical features corresponding to the $\chi_{R}$ basis representation that becomes a good pointer basis if $\rho_{\text {nuc }}\left(R, R^{\prime}\right)=0$. In this case, the $\chi_{R}$ basis representation is a pointer basis for the nuclei, and the classical properties associated to a given $\chi_{R}$ function are dynamically stable.

We have selected $\chi_{R}$ as a basis set over the nuclear configuration space (or more precisely, for practical purposes, a dense mesh of Dirac delta distributions over nuclear configurations), because we would like to study molecular structure and check whether the interference terms are suppressed between nuclear configurations. It is necessary to study whether and under which circumstances $\rho_{\text {nuc }}\left(R, R^{\prime}\right) \approx 0\left(R \neq R^{\prime}\right)$, i.e., the nuclear structure will be dynamically stable as a classical property.

a. Born-Oppenheimer molecular wave function It is interesting to note that all arguments are applicable not only for the (pre-Born-Oppenheimer) molecular wave function, but also for the conventional Born-Oppenheimer (BO) product of the electronic and nuclear wave functions, $\Psi^{[\mathrm{BO}]}(r, R)=\Psi_{\mathrm{el}}(r, R) \Psi_{\text {nuc }}(R)$. In this case, an off-diagonal element of the reduced density matrix is

$$
\begin{aligned}
\rho_{\mathrm{nuc}}^{[\mathrm{BO}]}\left(R, R^{\prime}\right) & =\left\langle\chi_{R}\left|\hat{\rho}_{\mathrm{nuc}}\right| \chi_{R^{\prime}}\right\rangle \\
& =\int \mathrm{d} r^{\prime \prime} \Psi_{\mathrm{el}}^{*}\left(r^{\prime \prime}, R^{\prime}\right) \Psi_{\mathrm{nuc}}^{*}\left(R^{\prime}\right) \Psi_{\mathrm{el}}\left(r^{\prime \prime}, R\right) \Psi_{\mathrm{nuc}}(R) \\
& =\left[\int \mathrm{d} r^{\prime \prime} \Psi_{\mathrm{el}}^{*}\left(r^{\prime \prime}, R^{\prime}\right) \Psi_{\mathrm{el}}\left(r^{\prime \prime}, R\right)\right] \Psi_{\mathrm{nuc}}^{*}\left(R^{\prime}\right) \Psi_{\mathrm{nuc}}(R) .
\end{aligned}
$$

Thus, the interference amplitude between the $R$ and $R^{\prime}$ nuclear structures depends on the overlap of the $\mathrm{BO}$ electronic wave functions corresponding to the $R$ and $R^{\prime}$ nuclear configurations.

b. All-particle molecular wave function The molecular wave function can be represented as linear combination of many-particle electron-nucleus basis functions, most commonly using variants of an explicitly correlated Gaussian basis set [1, $33-$ 35]. Electrons and nuclei are handled in such a 'pre-Born-Oppenheimer' or 'allparticle' treatment on an equal footing. The nuclear reduced density matrix is obtained by direct evaluation of the integral in Eq. (3). Due to the equivalent treatment 
of all particles, it is straightforward to calculate reduced density matrices corresponding to different kinds of partitioning of the molecule to a subset of $(a, b, c, \ldots)$ particles as the 'system' and the remaining $(z, y, x, \ldots)$ particles as the 'environment'. In the present work we use the electron-nucleus partitioning. 
(a)

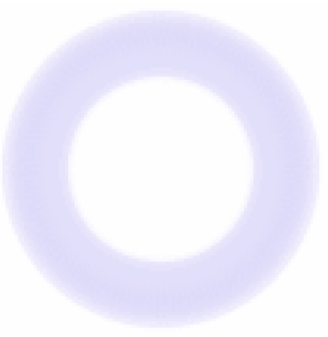

vs.

(b)

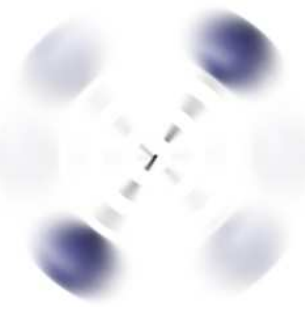

FIG. 2: Shell (a) or rotating dumbbell (b)? [E. Mátyus, Mol. Phys. 117, 590 (2019); licensed under a Creative Commons Attribution (CC BY) license.]

\section{ORIENTATIONAL LOCALIZATION: THEORETICAL FRAME- WORK}

For a start, let us consider a diatomic molecule in its ground rovibronic state with zero total angular momentum. This is a stationary state and the wave function is spherically symmetric. The one-particle nuclear density, $\rho_{0, \mathrm{n}}(R, R)$ calculated from this wave function shows that the nuclei ('n') are within a shell around the molecular center of mass, '0' (Fig. 1 of Ref. [25] and Fig. 2 of Ref. [26]). The two-particle density, $\rho_{0, \mathrm{nn}^{\prime}}(R, R)$, is strongly peaked at $180^{\circ}$ degrees for the included angle of the position vector of the two nuclei, $\mathrm{n}$ and $\mathrm{n}^{\prime}$, measured from the center of mass, '0' (Fig. 2 of Ref. [26]).

The one- and two-particle density functions describe well the internal nuclear structure but an additional question remains [1]: does the nuclear structure in the ground-state wave function resemble a shell with strong quantum coherences among the positions (Fig. $2 \mathrm{a}$ ) or a rotating dumbbell with a classical-like shape (Fig. 2b)?

We ask this question within the stationary-state, isolated molecule quantum treatment, because we would like to know whether this property is encoded in the molecular wave function itself, or it is induced by the environment. This question can be mathematically studied using the arguments and formalism developed in Sec. III,

So, we have to asses the orientational coherence or decoherence of the nuclei induced by the electrons. For this purpose, we consider nuclear configurations, $R$ and $R^{\prime}$ that have the same internal structure and are connected by spatial rotation, $R^{\prime}=\hat{O}_{\alpha} R$.

To quantify the interference between rotated structures, we will use the $\left\{\xi_{1}, \xi_{2}, \ldots\right\}$ basis set (Fig. 3) that consists of Dirac delta distributions over a dense 


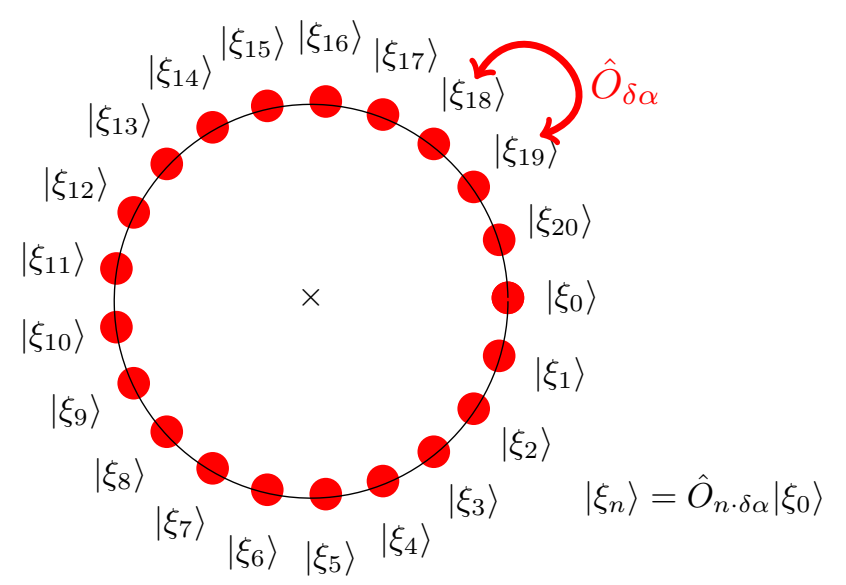

FIG. 3: Visual representation of the 'orientational basis' that we use to measure the emergence of the shape of the nuclear skeleton: Dirac delta distributions are located at molecular structures connected by rotation (shown in $2 \mathrm{D}$ ).

mesh in the configuration space of rotated nuclear structures, rather than a general $\chi_{R}$ configuration basis distributed over the entire configuration space (Sec. III). Hence, our measuring needles are $\xi_{\alpha}=\chi_{\hat{O}_{\alpha} R}$ (Fig. 3). So, in Eq. (44) we consider the rotational degrees of freedom for a selected $R$ nuclear structure (alternatively, the non-rotational part can be integrated out), and write the nuclear reduced density matrix operator corresponding to the rotated structures as

$$
\begin{aligned}
\hat{\rho}_{\mathrm{nuc}}^{\mathrm{rot}]}= & \int \mathrm{d} \alpha\left|\xi_{\alpha}\right\rangle\left\langle\xi_{\alpha}\right| \rho_{\mathrm{nuc}}\left(R_{\alpha}, R_{\alpha}\right) \\
& +\int \mathrm{d} \alpha \mathrm{d} \alpha^{\prime}\left|\xi_{\alpha}\right\rangle\left\langle\xi_{\alpha^{\prime}}\right|\left[1-\delta\left(\alpha-\alpha^{\prime}\right)\right] \rho_{\mathrm{nuc}}\left(R_{\alpha}, R_{\alpha^{\prime}}\right)
\end{aligned}
$$

where $\mathrm{d} \alpha$ collects the volume element for three Euler angles and the integrals are understood with the appropriate integration bounds. The $R_{\alpha}=\hat{O}_{\alpha} R$ is a short notation for the rotated structure.

If the off-diagonal elements of the nuclear reduced density matrix for the rotated structures $\left(R \neq R_{\alpha}\right)$,

$$
\rho_{\mathrm{nuc}}\left(R, R_{\alpha}\right)=\int \mathrm{d} r^{\prime \prime} \Psi\left(r^{\prime \prime}, R\right) \Psi^{*}\left(r^{\prime \prime}, R_{\alpha}\right)
$$


are small with respect to the diagonal elements,

$$
\rho_{\text {nuc }}(R, R)=\int \mathrm{d} r^{\prime \prime} \Psi\left(r^{\prime \prime}, R\right) \Psi^{*}\left(r^{\prime \prime}, R\right)
$$

then

$$
\hat{\rho}_{\text {nuc }}^{\text {[rot }]} \approx \int \mathrm{d} \alpha\left|\xi_{\alpha}\right\rangle\left\langle\xi_{\alpha}\right| \rho_{\text {nuc }}\left(R_{\alpha}, R_{\alpha}\right)
$$

i.e., the interference of the rotated nuclear structures is negligible. This nuclear reduced density matrix is mathematically identical with that of a classical mixture of rotated nuclear structures. This case corresponds to Fig. 1b in a diatomic molecule, the coherence of the rotated structures vanishes, and we may observe the emergence of a dumbbell-like shape of the nuclear skeleton. If the off-diagonal elements are large with respect to the diagonal ones, then there is a strong interference among the rotated nuclear structures and we cannot observe any new feature emerging beyond (within) the quantum mechanical rotational symmetry. In this case, the shell-like picture of Fig. 1a is appropriate. We note that both cases respect the original rotational symmetry of the molecular wave function. Either the classicallike or the quantum-like (as well as all intermediate) cases may be encoded within the molecular wave function depending on the strength of the interparticle interactions. The sole difference is in the suppression or existence of the coherence between pairs of rotated structures within the spherically symmetric molecular wave function.

When do the interference terms get small and the localization of the nuclei by the electrons efficient? It happens, if $\rho_{\text {nuc }}\left(R, R_{\alpha}\right)$, Eq. (8), is small. It is easier to understand the meaning of this condition within the Born-Oppenheimer approximation (when it is qualitatively correct), Eq. (6):

$$
\rho_{\text {nuc }}^{[\mathrm{BO}]}\left(R, \hat{O}_{\alpha} R\right)=\left[\int \mathrm{d} r^{\prime \prime} \Psi_{\mathrm{el}}\left(r^{\prime \prime}, R\right) \Psi_{\mathrm{el}}^{*}\left(r^{\prime \prime}, \hat{O}_{\alpha} R\right)\right] \Psi_{\mathrm{nuc}}(R) \Psi_{\mathrm{nuc}}^{*}\left(\hat{O}_{\alpha} R\right)
$$

If the overlap of the $\mathrm{BO}$ electronic wave functions corresponding to the rotated nuclear structures is small, then $\rho_{\text {nuc }}^{[\mathrm{BO}]}\left(R, R^{\prime}\right)$ is also small. 


\section{NUMERICAL STUDY OF ROTATIONAL LOCALIZATION OF THE NUCLEI IN THE MOLECULAR WAVE FUNCTION}

We have computed the ground-state wave function for four-particle systems, including the hydrogen molecule, $\mathrm{H}_{2}$, the positronium molecule, $\mathrm{Ps}_{2}$, the muonium molecule, $\mu_{2}$, and other $\mathrm{H}_{2}$-like systems, as well as $\mathrm{HeH}^{+}$, to illustrate the ideas formulated in the preceding sections.

The molecular wave function was computed using an explicitly correlated Gaussian basis set and the QUANTEN computer program 11]. In the present work, we use a plain ECG representation similarly to Refs. [25, 26] and the center-of-masscentered translationally invariant coordinates [1, 26, 35] to evaluate the diagonal and off-diagonal particle densities referenced to the molecular center of mass. We have converged the studied structural density features for the ground state of these systems with zero total angular momentum $(N=0)$, natural parity $(p=+1)$, and zero spin for the pair of electrons, protons, and positrons $\left(S_{\mathrm{e}}=0, S_{\mathrm{p}}=0\right)$. The corresponding energies were converged within ca. $1 \%$ that was sufficient to obtain density plots converged within the resolution of the figures.

To answer the question of Ref. [1] highlighted in Figure 2, we have calculated the off-diagonal elements of the nuclear reduced density matrix in the rotational basis (Sec. III),$\rho_{0, n}\left(R, \hat{O}_{\vartheta} R\right)$, where $\hat{O}_{\vartheta}$ is the operator for the rotation. The rotation axis goes through the molecular center of mass and $\vartheta$ parameterizes the rotation angle about this axis. Figure 4 shows the effect of the relative mass and electric charge on the orientational (de)coherence for a series of $\mathrm{H}_{2}$-type systems including $\mathrm{P}_{\mathrm{s}_{2}}, \mu_{2}$, the deuterium and tritium isotopologues of $\mathrm{H}_{2}$ and hypothetical (bound) systems with an increased nuclear charge.

We may observe in the figures that $\mathrm{P}_{\mathrm{s}_{2}}$ is qualitatively different from the other 'homonuclear' systems. Its diagonal 'nuclear' (positron) density, $\rho_{0, n}(R, R)$ has a maximum at the origin ( $R=0$ bohr $)$. Regarding the off-diagonal density, it shows a $360^{\circ}$ periodicity and has a small local maximum at $180^{\circ}\left( \pm i \cdot 360^{\circ}\right)$ and shallow local minima at $139^{\circ}$ and $221^{\circ}$ with a $1 \%$ suppression compared to the maximal (diagonal) value. 

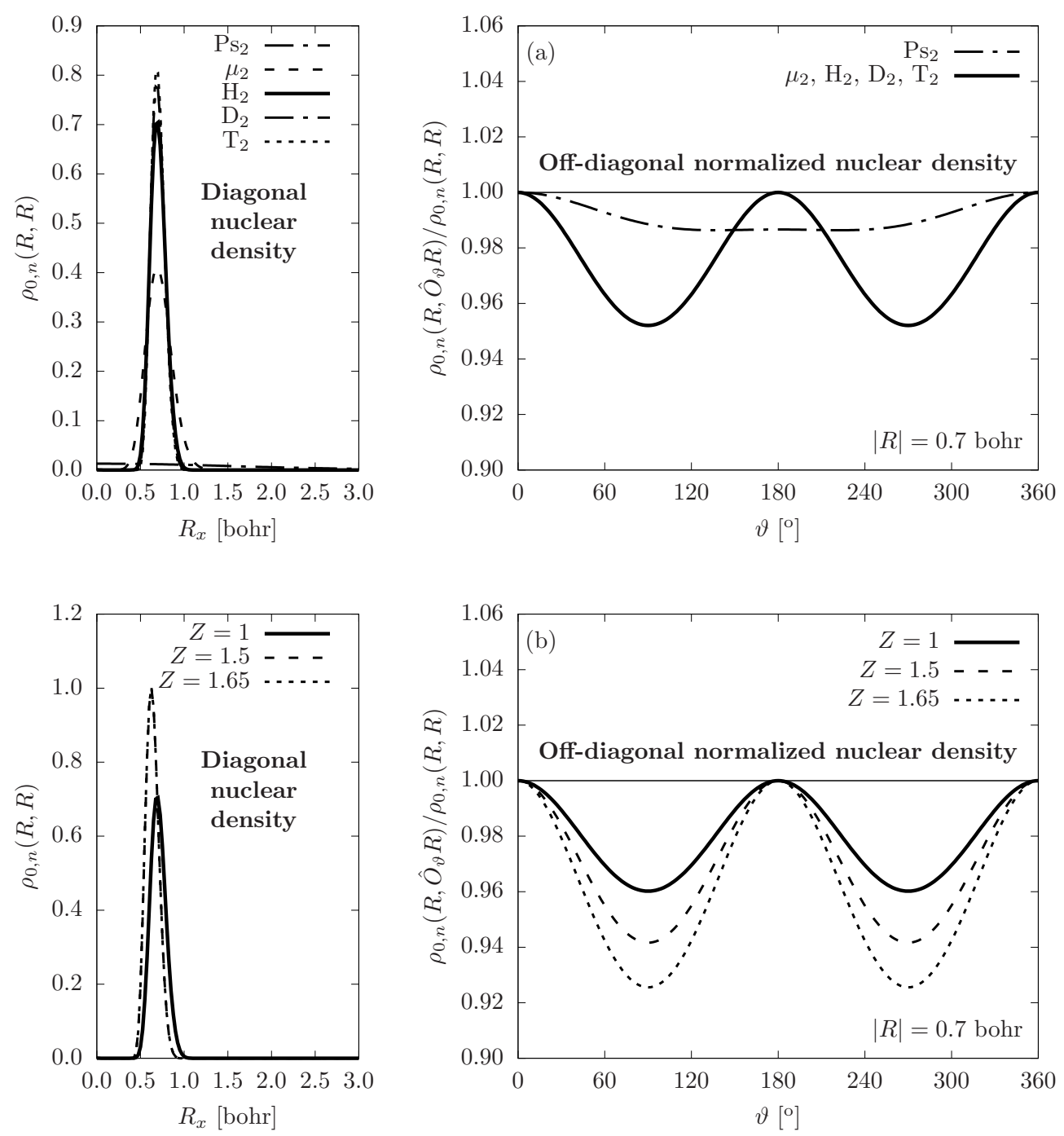

FIG. 4: Off-diagonal normalized density matrix elements connecting rotated nuclear structures in $\mathrm{H}_{2}$-type systems. The rotation axis for $\theta$ goes through the molecular center of mass that is the origin of the coordinate system used in the calculations. The diagonal density matrix elements are also plotted. (a) Effect of varying the mass of the positively-charged particles ('nuclei'), $m_{i} / m_{\mathrm{e}}=1,206.768,1836.15,3670.48$, and 5496.92 for $\mathrm{Ps}_{2}, \mu_{2}, \mathrm{H}_{2}, \mathrm{D}_{2}$, and $\mathrm{T}_{2}$, respectively. (b) Effect of varying the electric charge of the proton.

For $\mu_{2}, \mathrm{H}_{2}, \mathrm{D}_{2}$, and $\mathrm{T}_{2}$, the diagonal density functions differ but they all have a maximum (of different value for the different systems) near $|R|=0.7$ bohr. Within the resolution of the figure, the off-diagonal density matrix elements normalized with the diagonal density value (shown for $|R|=0.7$ bohr, Fig. 4a) cannot be distinguished for the four systems that may be surprising at first sight. We may understand this observation by remembering that the suppression is induced by the electrons' measuring effect that depends on the electronic structure around the positive particle 

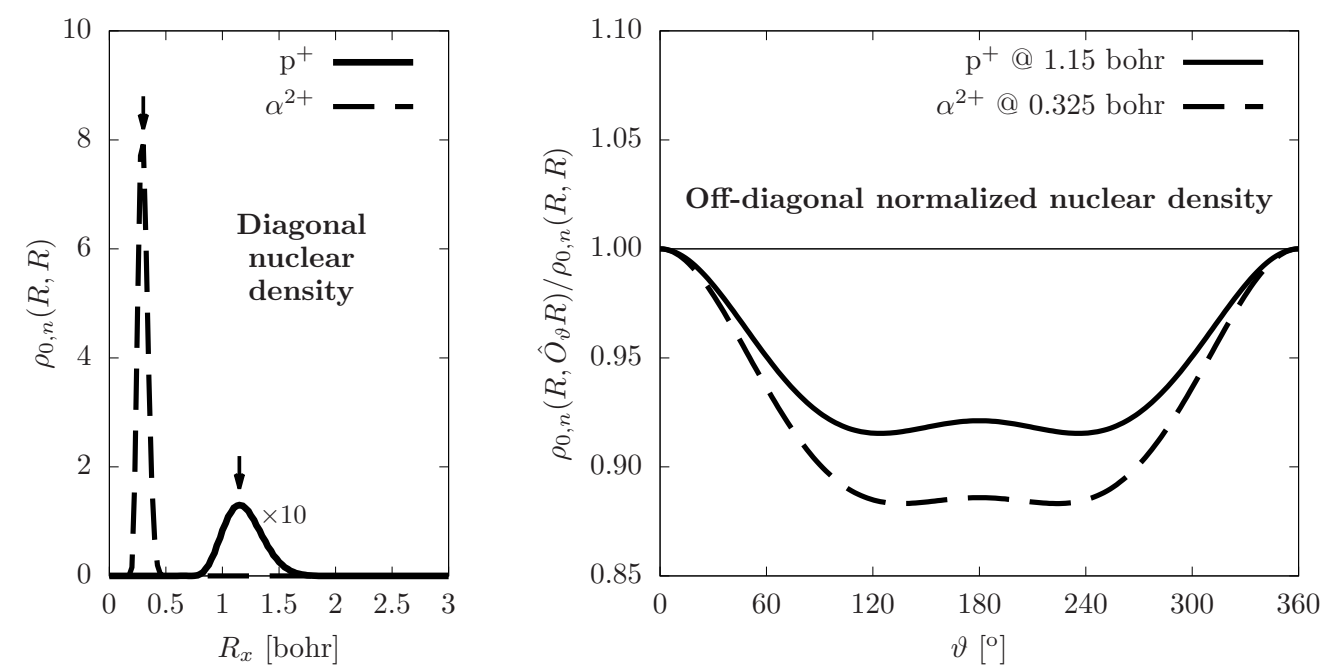

FIG. 5: Off-diagonal normalized nuclear density in $\mathrm{HeH}^{+}$connecting rotated nuclear structures. The diagonal nuclear density is also shown.

[Eq. (11)] and this is very similar in the four systems (the BO approximation is qualitatively correct). For this series of systems, the off-diagonal density shows a $180^{\circ}$ periodicity and it is minimal at $90^{\circ}\left( \pm i \cdot 180^{\circ}\right)$. Its minimum value is ca. $5 \%$ smaller than its maximal (diagonal) value.

Regarding the $\mathrm{H}_{2}$-like systems with an increased nuclear charge (Fig. 4b), we observe a behavior similar to $\mathrm{H}_{2}$, but a stronger, up to $8 \%$, suppression at the $90^{\circ}\left( \pm i \cdot 180^{\circ}\right)$ minima for $Z=1.65$. This observation is in agreement with the fact that the orientational de-coherence of the nuclei is induced by the monitoring effect of the electrons, and for an increased nuclear charge this monitoring effect is stronger due to the stronger interaction. (We note that the $\mathrm{H}_{2}$-like with $Z \geq 2$ systems are unbound.)

In summary, we may say that all $\mathrm{H}_{2}$ systems show dominant orientational coherences. $\mathrm{Ps}_{2}$ is an almost perfect quantum ball with an only $1 \%$ maximal suppression among the rotated structures. In the $\mathrm{H}_{2}$-like systems, the $180^{\circ}$ periodicity and maximal suppression at $90^{\circ}\left( \pm i \cdot 180^{\circ}\right)$ suggest a symmetric dumbbell shape (within the spherically symmetric molecular wave function), but due to the small value of the suppression ( $5 \%$ ), this dumbbell shape is blurred by quantum coherences.

$\mathrm{HeH}^{+}$is a heteronuclear diatomic molecule with an asymmetric dumbbell shape in the BO theory, while its rotational ground-state molecular wave function is, of course, spherically symmetric. The question arises whether this asymmetry can be 

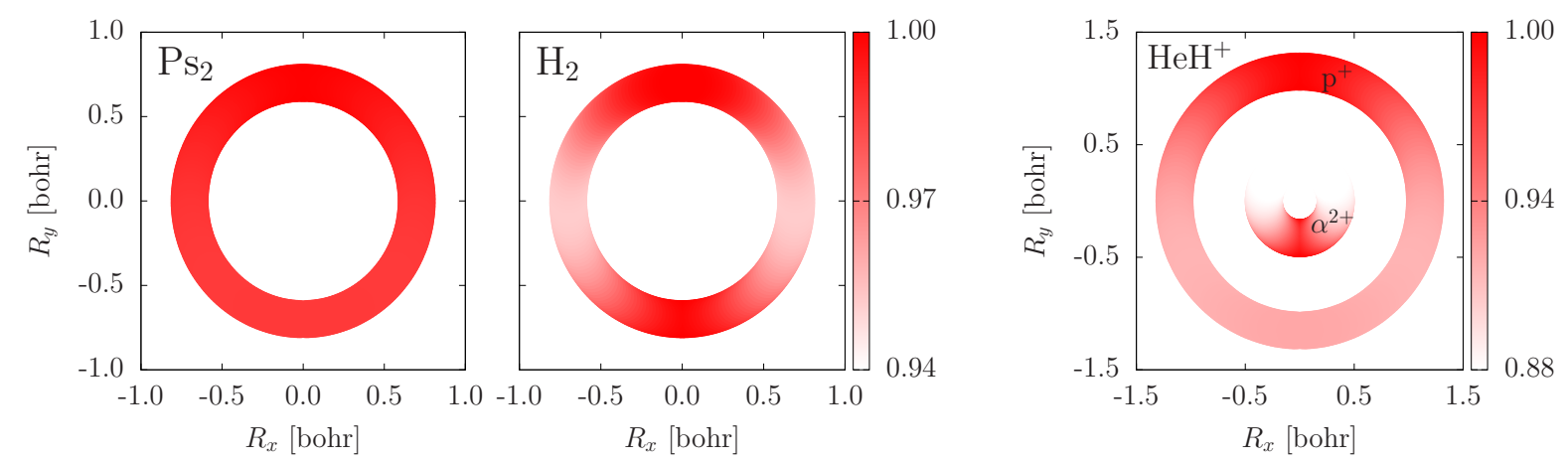

FIG. 6: Orientational (de)coherence measured by the suppression of the off-diagonal nuclear density with respect to the diagonal elements, $\rho_{0, n}\left(R, \hat{O}_{\vartheta} R\right) / \rho_{0, n}(R, R)$. The figure shows the $x y$ cut of the three-dimensional function (the width of the shells is arbitrarily chosen to visualize the change of the off-diagonal normalized density with the angle). The upward (downward) vertical direction correspond to zero rotation angle $\vartheta$ for the positron and the proton $(\alpha$-particle).

recognized within the spherically symmetric molecular wave function. Figure 5 shows the diagonal and off-diagonal nuclear density functions. The off-diagonal normalized density functions are plotted for $|R|$ values that correspond to the diagonal density maximum value for the proton and the alpha particles. The off-diagonal density function is periodic by $360^{\circ}$, it has equivalent minima at $\sim 120^{\circ}\left( \pm i \cdot 360^{\circ}\right)$ and $\sim 240^{\circ}\left( \pm i \cdot 360^{\circ}\right)$, and small local maxima at $180^{\circ}\left( \pm i \cdot 360^{\circ}\right)$ with an overall $10 \%$ suppression for $\vartheta \in[100,300]^{\circ}$ with respect to the maximal (diagonal) density value.

Figure 6] summarizes the three qualitatively different behavior observed in this work. $\mathrm{Ps}_{2}$ is an almost perfect quantum-ball with a maximal $1 \%$ suppression among rotated structures, $\mathrm{H}_{2}$ has a symmetric dumbbell shape, while $\mathrm{HeH}^{+}$has an asymmetric dumbbell shape, but both dumbbells are blurred by quantum coherence. 


\section{SUMMARY, CONCLUSION, AND OUTLOOK TO FUTURE WORK}

This work has been devoted to the study of the emergence of the classical molecular shape of an isolated molecule described by its ground-state rotation-vibrationelectronic wave function. To mathematically formulate this, at first sight, paradoxical problem, we have studied the orientational decoherence of the nuclear skeleton under the continuous monitoring effect of the electrons of the molecule, serving as the most natural environment for the nuclei. Orientational (de)coherence is measured by the off-diagonal nuclear reduced density matrix in the (direct) spatial representation.

If the reduced density matrix elements connecting rotated structures are small, then the nuclear reduced-density matrix is mathematically equivalent to the classical sum of rotated nuclear structures, and thus, we may say that the nuclei behave as if they formed a classical-like skeleton with a given shape that rotates in space. We have formulated the corresponding equations and computed the nuclear reduced density matrix elements over the nuclear configuration space for a series of $\mathrm{H}_{2}$-type (homonuclear) systems and for the heteronuclear $\mathrm{HeH}^{+}$.

We observe a small, $10 \%$ maximal suppression of the off-diagonal density connecting rotated nuclear structures (due to the measuring effect of the electrons), and thus we may say that $\mathrm{H}_{2}$ and $\mathrm{HeH}^{+}$have a symmetric and asymmetric dumbbell-like shape, respectively, that is blurred by strong quantum coherences. The decoherence effect in $\mathrm{Ps}_{2}$ is almost negligible and it is seen in this analysis as an almost perfect quantum ball.

We consider molecular rotation and the study of the emergence of the classical molecular shape (in the restricted sense of suppression of interferences), as a prototypical example for the broader problem of reconstruction of isomers, enantiomers, conformers, rotamers, etc. from the molecular wave function (including the electrons and nuclei on an equal footing). Isomerism, handedness, and related phenomena are linked to polyatomic molecules, while already the smallest diatomic molecules have rotational degrees of freedom and they are attached with the picture of a classical shape in chemistry.

The present framework uses a spatial basis for the representation of the nuclear reduced density matrix that can naturally be adapted for studying other space- 
localized features. Regarding another challenging aspect of the molecular structure problem, the study of the quantum mechanical indistinguishability vs. the classical distinguishabilty of the identical atomic nuclei and implications of the spin-statistics theorem [36, 37] will require perhaps a different framework, but almost certainly a different representation for the reduced density matrix.

The developed ideas will gain more practical significance, if computations without the Born-Oppenheimer approximation become more widespread, which may happen within a new type of quantum chemistry approach perhaps on the future hardware of quantum computers [38].

Regarding work for the nearer future. We can think about extension of the present work in the following directions. A next logical step will be to recover the (2dimensional) triangular shape of the simplest polyatomic molecule, $\mathrm{H}_{3}^{+}$that has an equilateral triangular equilibrium structure in the Born-Oppenheimer theory. $\mathrm{H}_{3}^{+}$is a system of five spin- $1 / 2$ particles (with 2 electrons and 3 protons). For this reason, the rovibronic ground state of the five-particle Hamiltonian is not allowed by the Pauli principle. The lowest energy state that is Pauli allowed has $N=1$ rotational angular momentum, it is the lowest rotationally excited state of the vibrational ground state. The lowest Pauli-allowed state with $N=0$ rotational angular momentum is the anti-symmetric stretching fundamental vibration. So, the lowest energy $N=1$ state appears to be a good candidate for identifying a near equilateral triangular shape (with small distortion due to rovibrational coupling) in the present framework.

We have preliminary results for the ground state of $\mathrm{H}_{2} \mathrm{D}^{+}$, for which we do not have to deal with complications due to spin statistics, and in its rovibronic ground state $(N=0)$, we observe a ca. maximal $10 \%$ suppression among rotated structures. We can observe a planar structure in the off-diagonal density plots, but due to the non-equivalent nuclear masses the center of mass (the origin of our computations) is not at the geometrical center of the near equilateral triangular shape and this complicates the analysis of the results.

For going beyond planar shapes and possibly studying chiral molecules, one can rely on the BO approximation (Eq. (6) ), since the suppression effect is determined by the electronic structure. If the BO approximation is qualitatively correct for the 
system, then the overlap of the rotated BO electronic wave functions should provide a good approximation for the suppression effect that one would observe for the off-diagonal nuclear reduced density matrix in the molecular wave function (if the computations were feasible).

Data Availability Statement The data that support findings of this study is included in the paper.

Acknowledgement This project was initiated during a Short Term Scientific Mission of the MOLIM COST Action. EM acknowledges financial support from a PROMYS Grant (no. IZ11Z0_166525) of the Swiss National Science Foundation. We thank one of the Reviewers of this article for their insightful comments.

[1] E. Mátyus, Mol. Phys. 117, 590 (2019).

[2] J. Demaison, J. E. Boggs, and A. G. Csaszar, eds., Chemistry, Quantum Mechanics and Reductionism: Perspectives in Theoretical Chemistry (CRC Press, Boca Raton, FL, 2010).

[3] R. G. Woolley, Adv. Phys. 25, 27 (1976).

[4] R. G. Woolley and B. T. Sutcliffe, Chem. Phys. Lett. 45, 393 (1977).

[5] P. Claverie and S. Diner, Isr. J. Chem. 19, 54 (1980).

[6] H. Primas, Chemistry, Quantum Mechanics and Reductionism: Perspectives in Theoretical Chemistry (Springer-Verlag, Berlin, 1981).

[7] S. J. Weininger, J. Chem. Educ. 61, 939 (1984).

[8] P.-O. Löwdin, Pure and Appl. Chem. 61, 2065 (1989).

[9] B. T. Sutcliffe and R. G. Woolley, Phys. Chem. Chem. Phys. 7, 3664 (2005).

[10] E. Joos, H. Zeh, C. Kiefer, D. Giulini, J. Kupsch, and I. Stamatescu, eds., Decoherence and the Appearance of a Classical World in Quantum Theory, 2nd edition (SpringerVerlag, Berlin Heidelberg, 1996, 2003).

[11] M. Schlosshauer, Decoherence and the Quantum-to-Classical Transition (Springer, Berlin, 2007). 
[12] S. Fortin, O. Lombardi, and J. C. M. González, Found. Chem. 18, 225 (2016).

[13] G. Bacciagaluppi, "The Role of Decoherence in Quantum Mechanics", The Stanford Encyclopedia of Philosophy (Fall 2016 Edition), Edward N. Zalta (ed.), URL = https://plato.stanford.edu/archives/fall2016/entries/qm-decoherence/.

[14] P. Pfeifer, Chiral Molecules: A Superselection Rule Induced by the Radiation Field, PhD thesis ETH Zürich, Nr. 6551, ok Gotthard S + D AG, Zürich (1980).

[15] P. Pfeifer, A nonlinear Schrödinger equation yielding the 'Shape of molecules' by symmetry breaking, p. 255-266 in Quantum Mechanics in Mathematics, Chemistry and Physics, eds., K. E. Gustafson and W. P. Reinhardt (Plenum Press, New York, 1981).

[16] R. G. Woolley, Structure and Bonding (Springer) 52, 1 (1982).

[17] A. S. Wightman, Il Nuov. Cim. 110B, 751 (1995).

[18] P. Claverie and G. Jona-Lasinio, Phys. Rev. A 33, 2245 (1986).

[19] G. Jona-Lasinio and P. Claverie, Progr. Theor. Phys. Suppl. 86, 54 (1986).

[20] E. Joos and H. D. Zeh, Z. Phys. B 59, 223 (1985).

[21] K. Hornberger, Eur. Phys. Lett. 77, 50007 (2007).

[22] M. Busse and K. Hornberger, 43, 015303 (2009).

[23] J. Trost and K. Hornberger, Phys. Rev. Lett. 103, 023202 (2009).

[24] C. Zhong and F. Robicheaux, Phys. Rev. A 94, 052109 (2016).

[25] E. Mátyus, J. Hutter, U. Müller-Herold, and M. Reiher, Phys. Rev. A 83, 052512 (2011).

[26] E. Mátyus, J. Hutter, U. Müller-Herold, and M. Reiher, J. Chem. Phys. 135, 204302 (2011).

[27] E. V. Ludeña, L. Echevarría, X. Lopez, and J. M. Ugalde, J. Chem. Phys. 136, 084103 (2012).

[28] M. Becerra, V. Posligua, and E. V. Ludeña, Int. J. Quant. Chem. 113, 1584 (2013).

[29] E. V. Ludeña, P. Iza, M. Cornejo, and D. Zambrano, Theor. Chim. Acta 135, 76 (2016).

[30] A. Schild, Front. Chem. 7, 424 (2019).

[31] J. von Neumann, Mathematical Foundations of Quantum Mechanics (Princeton University Press, Princeton, NJ, 1955).

[32] W. H. Zurek, Phys. Rev. D 24, 1516 (1981). 
[33] J. Mitroy, S. Bubin, W. Horiuchi, Y. Suzuki, L. Adamowicz, W. Cencek, K. Szalewicz, J. Komasa, D. Blume, and K. Varga, Rev. Mod. Phys. 85, 693 (2013).

[34] S. Bubin, M. Pavanello, W.-C. Tung, K. L. Sharkey, and L. Adamowicz, Chem. Rev. 113, 36 (2013).

[35] E. Mátyus and M. Reiher, J. Chem. Phys. 137, 024104 (2012).

[36] T. Grohmann and J. Manz, Mol. Phys. 116, 2538 (2018).

[37] F. Bouakline, J. Chem. Phys. 152, 244308 (2020).

[38] I. Kassal, S. P. Jordan, P. J. Love, M. Mohseni, and A. Aspuru-Guzik, Proc. Nat. Acad. Sci. USA 105, 18681 (2008). 